\title{
An Airborne Parachute Compartment Test Bed for the Orion Parachute Test Program
}

\author{
James W. Moore* \\ Sierra Nevada Corporation, Louisville, CO, 80027, USA \\ Leah M. Romero ${ }^{\dagger}$ \\ Jacobs Engineering, Houston, TX, 77058, USA
}

\begin{abstract}
The test program developing parachutes for the Orion/MPCV includes drop tests with parachutes deployed from an Orion-like parachute compartment at a wide range of dynamic pressures. Aircraft and altitude constraints precluded the use of an Orion boilerplate capsule for several test points. Therefore, a dart-shaped test vehicle with a hi-fidelity mockup of the Orion parachute compartment has been developed. The available aircraft options imposed constraints on the test vehicle development and concept of operations. Delivery of this test vehicle to the desired velocity, altitude, and orientation required for the test is a difficult problem involving multiple engineering disciplines. This paper describes the development of the test technique. The engineering challenges include extraction from an aircraft, reposition of the extraction parachute, and mid-air separation of two vehicles, neither of which has an active attitude control system. The desired separation behavior is achieved by precisely controlling the release point using on-board monitoring of the motion. The design of the test vehicle is also described. The trajectory simulations and other analyses used to develop this technique and predict the behavior of the test vehicle are reviewed in detail. The application of the technique on several successful drop tests is summarized.
\end{abstract}

\section{Nomenclature}

\section{$C_{D} S \quad$ Drag Area}

\section{Introduction}

$\mathrm{T}$ HE Capsule Parachute Assembly System (CPAS) project is tasked with developing the parachute system for the Orion/MPCV command module. CPAS includes a pair of mortar-deployed drogue parachutes intended to decelerate and stabilize the capsule prior to deployment of the main parachute system. The three main parachutes are deployed by a set of three pilot parachutes which themselves are independently mortar-deployed. A detailed description of the entire parachute system and concept of operations can be found in Machin, et. al. ${ }^{1}$ The CPAS project has developed a variety of test techniques to evaluate and qualify this system. ${ }^{2}$ The technique described here is a new addition to this collection. A critical component of the test plan is the testing of the mid-air deployment of the parachute system from a realistic parachute compartment at a wide range of dynamic pressures. The test vehicle designed to achieve this goal is a dartshaped body fitted with a hi-fidelity mock-up of the Orion parachute compartment named the Parachute Compartment Drop Test Vehicle (PCDTV). The options for delivering this type of vehicle to the required altitude are limited. ${ }^{3}$ C-130 extractions were selected due to cost and schedule availability. This paper describes the development of this vehicle and the engineering task of successfully delivering it to the desired test conditions.

*Engineer, Space Systems Group, 1722 Boxelder St., Louisville, CO, AIAA Member.

${ }^{\dagger}$ Analysis Engineer, Aeroscience and Flight Dynamics, 2224 Bay Area Blvd, Houston, TX, AIAA Member. 
The goals and constraints for the design of the test technique and the resulting PCDTV will be described. The vehicle design was subject to significant geometrical constraints that required trade-offs between aerodynamic and structural concerns. The extraction of the test article from an aircraft required the use of a test support structure called the Mid-air Delivery System (MDS) that acts as an interface between the aircraft and the test vehicle. The test vehicle must be separated from this structure after extraction and before the test point is achieved. The Concept of Operations for this sequence will be described. Design of the trajectory sequence of events and prediction of the test article motion required the development of a new simulation model, enhancement of existing simulations, and new methods for integrating various analysis tools to understand the end-to-end performance of the tests. The toolset used to perform these analyses will be outlined with particular attention to the interface between various tools. The engineers performed a variety of pre-flight analyses to ensure the success of the test. Several of the more important analyses will be outlined. Finally, the deployment of the technique on several tests will be summarized.

\section{Description of the Test Vehicle and Techniques}

The PCDTV was designed to provide realistic parachute deployments in drop tests that target high dynamic pressures. The development of the vehicle and the concept of operations will be described below. Aircraft and structural constraints forced compromises with the aerodynamic goals. The important trade-offs will be highlighted.

\section{A. Concept of Operations}

The extraction of PCDTV from the aircraft and separation from the MDS is modeled after the successful Ares parachute program "Jumbo Dart" series. ${ }^{4}$ Figure 1 is a schematic of the PCDTV test Concept of Operations. The mated PCDTV/MDS system is extracted from a C-130A using a modified Low Velocity Air Delivery (LVAD) technique with a shortly delayed load transfer from the Extraction Force Transfer Coupling (EFTC). Just prior to the separation of the PCDTV and MDS, the extraction chute(s) reposition from a single attach point (EFTC) at the rear of the MDS to a four point harness attached near the corners of the MDS. The reposition event is executed by a timer that is set based on pre-flight analysis. The reposition to the inverted sling induces a pitch-over so that the PCDTV is travelling in-front-of rather that on-top-

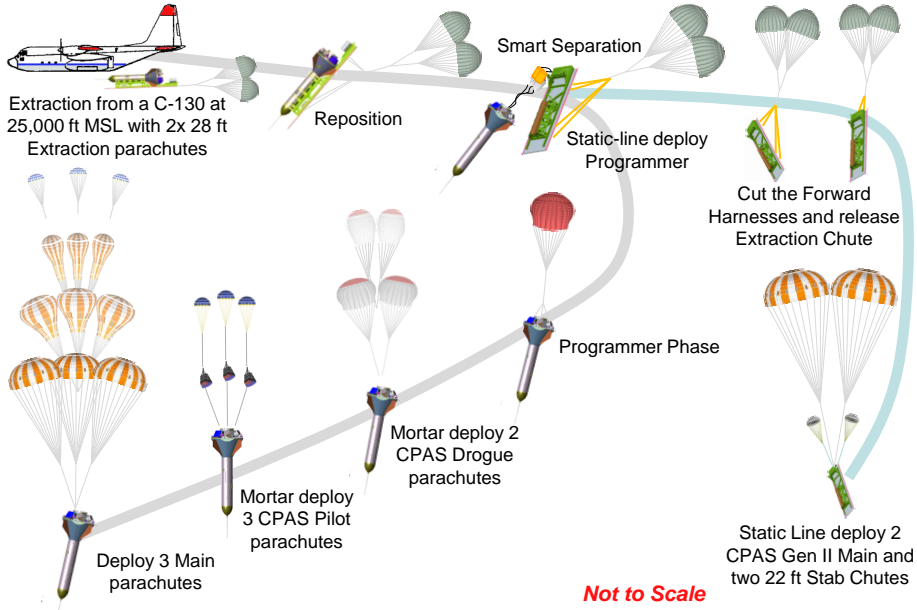

Figure 1. Typical PCDTV Test Concept of Operations. of the MDS relative to the airstream. A Smart Separation routine commands the pyrotechnic cut of the straps holding the PCDTV to the MDS. CPAS analysts design the separation event to minimize the likelihood of re-contact. The programmer parachute is static-line-deployed from the PCDTV using a deployment line attached to the MDS. At a pre-determined time (when predictions show the test point will be achieved) the onboard avionics commands the cut-away of the programmer and the CPAS Drogue parachutes are mortar-fired. After the Drogue parachutes perform the scheduled disreefing they too are cut and the pilot chutes are mortar-fired. The pilots in turn pull out the CPAS Main parachutes. The PCDTV descends to the ground under the Main parachutes which perform a planned reefing schedule. All parachute cut and mortar-fire events are based on time and are established with pre-flight analysis. The MDS descends under the extraction chute system until two CPAS Mains and two stabilizer parachutes are deployed to slow the rate-of-descent to acceptable levels. The stabilizer parachutes are intended to maintain attitude control of the MDS while the Main parachutes inflate. 


\section{B. Test Vehicle Design}

There were three primary goals in the design of the PCDTV and the test technique. First, the vehicle should have a hi-fidelity representation of the Orion parachute compartment. Second, the tests should be able to achieve a high dynamic pressure. Third the test vehicle should be stable and fly predictably. A capsuleshaped test vehicle satisfies the first desire but presents significant challenges for achieving the latter two goals. The PCDTV has a slender forebody to achieve high dynamic pressure. A full-size Orion parachute compartment is mounted on the aft end. Fins and nose-ballast are included to improve stability. The PCDTV is shown in Fig. 2.

\section{Aircraft Constraints}

The CPAS project had performed numerous drop tests with dart shaped test vehicles prior to the start of the PCDTV test series. These tests employed smaller missile shapes with diameters of 2 feet or less. Those vehicles were either dropped from a vertical orientation under a helicopter or extracted in a horizontal orientation from a C-130. The concept of operations for the horizontal extraction tests were similar to the ConOps envisioned for the PCDTV series. The availability of C-130A aircraft made this airframe highly attractive option for the PCDTV series. However, the diameter of Orion parachute compartment is too large to pass through the C-130 aft door. Truncating the bottom of the parachute compartment so that two opposite edges were flat (rather than circular) allowed the parachute compartment to fit through the C-130 door in a without significantly

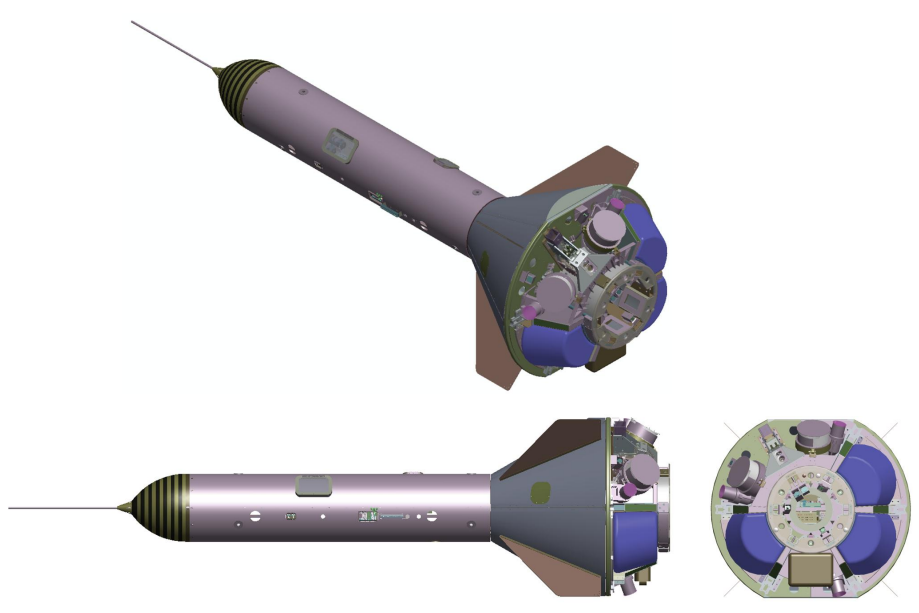
affecting the rigging or deployment of the parachutes.

\section{Attach Point Configurations}

The PCDTV parachute compartment has 6 attach locations evenly spaced around the top of the parachute compartment tunnel. These attach points are intended to be used in the programmer phase of the test. The main and drogue parachutes are attached through the parachute compartment as they will be in the operational vehicle. Test engineers are free to select the programmer attach points as needed to achieve test objectives. The goal of the programmer phase is to deliver the PCDTV to the desired test dynamic pressure and to damp out any residual pitch or yaw motion resulting from the separation event. A four point harness is typically employed to attach the programmer at evenly spaced attach points around the tunnel.

\section{Release Mechanism}

The prior CPAS dart-shaped tests used a Cradle Monorail System (CMS) as an interface between the test vehicle and the aircraft. The CMS included a rail that guided the separation of the dart. The shape of the PCDTV, with a cylindrical fuselage and a large flared tail made the application of a rail impractical. The CPAS project elected to build a custom cradle to hold the cylindrical part of the PCDTV and adopt the separation technique proven in the Ares program Jumbo Dart test series. The PCDTV is fixed to the MDS with a set of straps that pass over the cylindrical section. At the commanded release condition, these straps are cut. To avoid recontact, the mated vehicle must be properly oriented at the time of separation. This required the inclusion of an extraction parachute reposition event in the test technique. 


\section{Test Technique}

The PCDTV Concept of Operations included two events that were outside of previous CPAS experience. CPAS had not performed a reposition to the underside of a Type V platform in any previous test. Also, midair separations of dart-like vehicles on previous tests had employed the Cradle Monorail System (CMS) to guide the separation of the dart. The following section provides more detail on the new techniques employed in the the PCDTV series of tests.

\section{Reposition}

Previous CPAS dart tests differed significantly in the separation technique. In tests using the CMS, the test vehicle slides forward along a rail. The direction of separation is parallel to the test vehicle axis of symmetry. The boat-tail shape of the PCDTV made the attachment of a rail problematic. Instead, the CPAS project opted for a separation similar to the Ares Jumbo Dart tests. In this type of separation, the dart lifts off of the cradle system and the direction of separation is perpendicular to the test article axis of symmetry. This necessitates a reposition of the extraction parachute so that the momentum of the dart vehicle and the drag on the cradle are roughly perpendicular to the dart axis of symmetry. After the reposition, the extraction harness is attached ot the underside of the Type $\mathrm{V}$ pallet. The timing of this repostion and the geometry of the inverted harness was the subject of much of the initial pre-flight analysis.

\section{Smart Separation}

The CPAS project developed a Smart Separation system in anticipation of this and other test techniques. ${ }^{5}$ Preliminary analysis indicated that the attitude and attitude rates at the separation event would need to be constrained in order to avoid re-contact as the PCDTV leaves the MDS cradle. Moreover, structural concerns limited the magnitude of the nose ballast which resulted in the center-of-gravity being less forward than desired and mass concentrations on either end of the vehicle. This dumbbell-like set of mass properties increased the tendency to pitch toward a more parachute-compartment forward orientation when the separation rates were large. Analysis showed that the PCDTV would recover from this orientation but the programmer harness was likely to wrap over the parachute compartment increasing the chance of entanglement and severed lines. The Smart Separation algorithm was employed to avoid this outcome.

\section{Toolset Capabilities and Limitations}

CPAS analysts used three simulation to design test trajectories and compute loads during the development of the PCDTV. The use of multiple trajectory simulations was required because none of the available simulations included all the necessary capabilities. The interface between the various simulations is performed with a suite of MATLAB and Python scripts. Each tool is described here and the strengths and limitations are noted.

\section{A. Decelerator System Simulation (DSS)}

The Decelerator System Simulation ${ }^{6}$ (DSS) is a legacy six degree-of-freedom (6-DOF) parachute trajectory simulation based on the UD233A ${ }^{7}$ simulation used by the Space Shuttle Solid Rocket Booster parachute project. DSS is written in the Fortran programming language and user input is provided via text files. DSS is the highest-fidelity, NASA-maintained simulation used by the CPAS project. However, DSS does not model contact forces between multiple bodies and is, therefore, not suitable for analysis of the extraction phase or the PCDTV/MDS separation.

\section{B. Decelerator System Simulation Application (DSSA)}

The Decelerator System Simulation Application ${ }^{8}$ (DSSA) is a 6-DOF parachute trajectory simulation based on DSS that includes the capability to model the extraction of a test platform from an aircraft. Like DSS, this simulation is written in Fortran. All previous CPAS aircraft extractions have been analyzed with DSSA. DSSA does not model the interaction of the vehicles during the separation nor is it well-suited to model the change in mass properties and aerodynamics that occurs when the test article is separated from the MDS. 


\section{Adams}

MSC/Adams is a commercial multi-body 6-DOF simulation. None of the CPAS simulations mentioned above include hi-fidelity multi-body capability suitable for analyzing the separation of the PCDTV from the MDS. Adams was used to fill this gap. The Adams model developed for these studies included contact forces with the aircraft floor and a simplified extraction parachute drag area, $C_{D} S$, growth curve. This provided significant overlap with DSSA and several sim compare studies were performed to verify the Adams model. A hi-fidelity parachute inflation model was not added to Adams so the portion of the test from separation to ground was simulated in DSS.

\section{Load Train Analysis}

Analysts calculated the loads developed during the static-line deployment of the programmer using a set of spreadsheet and MATLAB tools. The spreadsheet methods compute the instantaneous change in velocity at the snatch events and convert this into the energy to be absorbed by the soft goods. The forces are then computed from this absorbed energy result by treating the soft goods as linear springs. Engineers also developed a MATLAB 2-DOF deployment simulation that directly integrates the equations of motion for the point masses between the soft goods. This tool was used to cross-check the spreadsheet results.

\section{Analysis Techniques}

The PCDTV series of tests required the development of several analysis techniques not previously used in CPAS testing. The majority of the analysis was concerned with the early portion of the test, including the extraction, reposition, separation, and programmer deployment. As described above, the reposition and separation techniques were new to the CPAS drop test program. CPAS had not performed the inverted reposition in any previous test. Previous mid-air separations of dart-like vehicles had been controlled with a monorail system. The following section describes the new analysis techniques employed to bring the PCDTV system to test readiness.

The analysis was primarily concerned with the pitch plane motion since this dominates the dynamics after the aircraft ramp is cleared. Figure 3 shows typical motion in pitch, pitch rate, and angle-of-attack immediately after extraction. Because of the oscillation, the free-
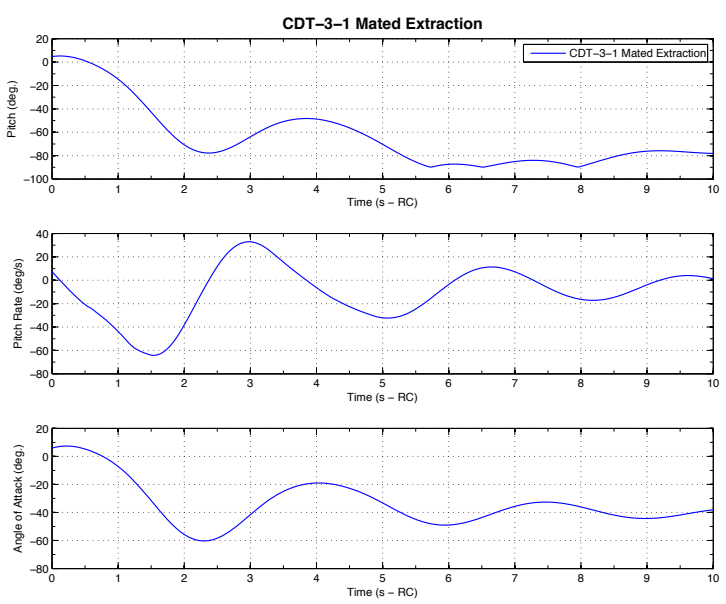

Figure 3. Pitch plane motion of the mated vehicle after extraction

flight motion of the PCDTV is sensitive to the instant of separation from the MDS. The overall goal of all the analyses was to understand the motion of the mated vehicle in the first few seconds after extraction and to identify the separation conditions that would avoid recontact between the PCDTV and MDS and minimize rotation of the PCDTV. The portion of the test from the programmer deployment through touchdown was similar to other CPAS tests and was analyzed with Monte Carlo trajectory simulation with the DSS tool.

\section{A. Reposition Event}

Several types of analyses were related to designing the reposition event. These included, the selection of the reposition harness geometry, the timing of the reposition event, and evaluation of the loads developed by the reposition. Along with the mass properties and the extraction parachute forces, the timing of the reposition and the geometry of the reposition harness are major factors in determining the subsequent motion of the mated vehicle. These analyses will be described below. 


\section{Sling Configuration}

The reposition event moved the extraction parachute attach point from the EFTC fitting to a four point harness attached near each corner of the Type V pallet. The DSSA simulation included the capability to model a reposition but the existing model would not accommodate the inverted harness. The simulation had to be modified to correctly compute the resulting forces.

A design of experiments approach was employed to simultaneously assess several potential sling geometries as well as the separation time. The potential configurations were evaluated using the resulting orientation of the PCDTV at separation. This analysis was one of the first to be performed and a multibody simulation was not yet part of the toolset. To make progress, the separation was assumed to take place instantaneously.

The potential for recontact was assessed by comparing the direction of motion at the instant of separation to the geometry of the two vehicles. Specifically, the angle of attack was compared to the angle of the PCDTV flare. This angle of roughly $24 \mathrm{deg}$ is shown in the top diagram in Fig. 4. If it is also assumed that the extraction parachute aligns itself so that the riser is parallel to the velocity vector then the MDS can be expected to be drawn away from the PCDTV along the negative velocity vector. With these assumptions, comparison of the angle of attack to the angle between the flare and the MDS cradle gives a rough indication of the orientations that will avoid recontact. Of course, this analysis
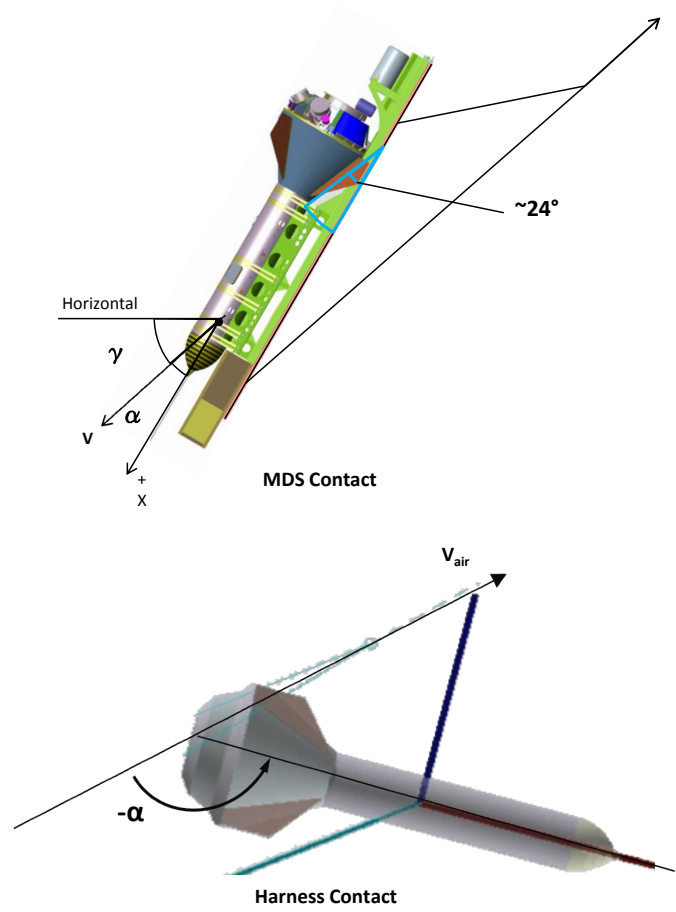

neglects any relative rotation between the PCDTV and MDS, but it was suitable to identify feasible harness configurations.

The analysis indicated that it was possible to provide sufficient negative pitch angle through the harness geometry such that the release pitch rate could be targeted for zero. This significantly simplified the follow on analysis since it meant the PCDTV could be delivered to the desired orientation and then separated with essentially no relative angular rate. The PTV test series that coincides with the PCTDV test series did not have this luxury. ${ }^{9}$ The PTV separates at an orientation that is not the desired final orientation. An appropriate amount of pitch rate is required to achieve the desired orientation in free flight. The harness lengths chosen for the first PCDTV test were $34 \mathrm{ft}$ for the front sling legs and $14 \mathrm{ft}$ for the aft sling legs. This initial study indicated that these lengths were feasible and would allow the mated vehicle to pass through the desired separation conditions.

\section{Reposition Timing Constraints}

An analysis was required to determine an appropriate time to initiate the reposition of the extraction parachute from the EFTC to the four point harness beneath the MDS. There were two competing factors at play. First there is a desire to delay the reposition until the mated vehicle is sufficiently clear from the aircraft. On the other hand, there was some evidence suggesting that, because of the delayed load transfer in this modified LVAD technique, the EFTC could bind and fail to release if it were subject to bending against the hardstop that prevents rotation below the Type $\mathrm{V}$ pallet (a standard LVAD technique releases the EFTC at ramp clear).

With the delayed EFTC release, as the extracted vehicle clears the ramp, the forward edge tends to dip down past the already falling aft edge (resulting in a negative pitch attitude) and initiating a pitch 
oscillation. Since the extraction parachute riser could be assumed to be roughly horizontal at the time that the mated vehicle clears the aircraft ramp, the PCDTV/MDS pitch gives a rough indication of the angle that the EFTC makes with the pallet. DSS Monte Carlo simulations were performed to identify the earliest time that the mated vehicle pitch would become negative (thus potentially binding the EFTC). The analysis resulted in a reposition time of 0.5 seconds for the first PCDTV test.

\section{B. Constraints on Separation Timing}

The previous analyses were important in determining the motion of the mated vehicle during and after the reposition. Once the reposition time and harness geometry design decisions were made, the CPAS analysts could use the existing simulation tools to examine the motion and determine the optimum conditions for separation. The engineers identified two limiting cases that helped to constrain the problem. Although the discriminating factors were the pitch and pitch rate, the motion followed a specific pattern so it became convenient to think of the limiting cases as separation events that were either too early or too late.

\section{Early Separation}

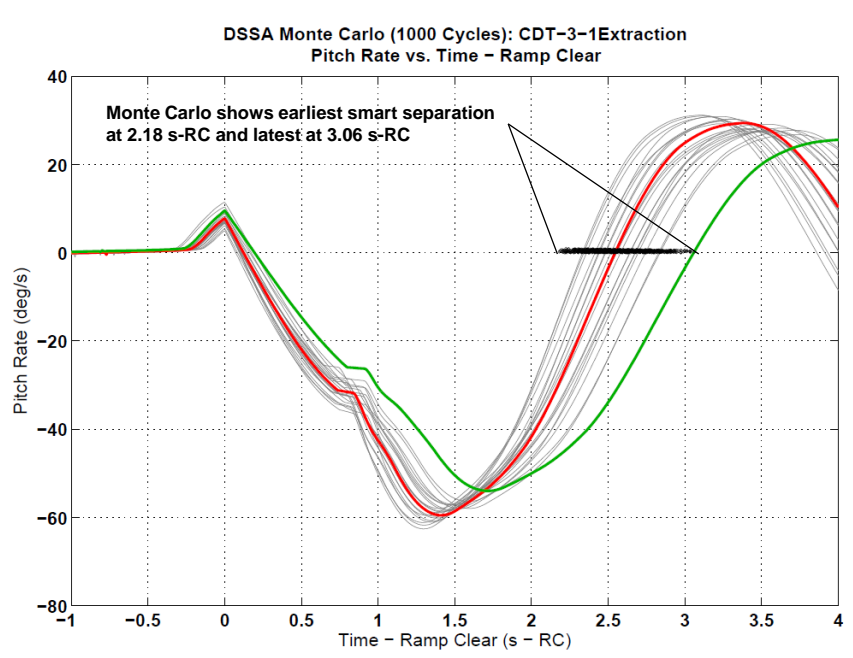

The overall motion of the mated vehicle and parachute system immediately after clearing the ramp is a downward swing of the mated vehicle under the parachute. Superimposed on this motion is a pitch down of the PCDTV/MDS as the reposition occurs. The aft end of the PCDTV/MDS is suddenly allowed to rotate over the top of the forward end. During this early part of this motion the PCDTV is still pointed toward the direction of travel which is roughly horizontal. At this point the PCDTV has a low negative pitch angle and a small angle of attack. If the separation occurs in this attitude then the PCDTV will move roughly parallel to the MDS. In this case there is a risk that the flared end of the PCDTV will contact the cradle portion of the MDS. This situation is shown in the top diagram in Fig. 4. To avoid this condition, the engineers sought to maintain the angle of attack at separation to be less than -45 degrees.

\section{Late Separation}

As described above, after the reposition the aft end of the mated vehicle tends to rotate forward and over the forward end. Simultaneously the mated vehicle is achieving an increasingly negative pitch angle and angleof-attack. For separation at large pitch rates or large negative pitch angles, the parachute compartment will tend to continue this motion and rotate over the nose. In other words, the PCDTV will rotate away from the MDS rather than translate away. These higher rates and lower angles occur later in the motion.

The programmer parachute system is attached to the parachute compartment at the aft end of the PCDTV. The programmer deployment bags are attached to the MDS and the programmer system is static line deployed as the PCDTV moves away from the MDS. If the rotation of the PCDTV is sufficiently large, the programmer riser may come in contact with the parachute compartment and risk becoming severed. To avoid this situation, the analysts sought to avoid post separation angles-of-attack less than -90 deg. This situation is shown in the bottom diagram in Fig. 4. Violations of this constraint were less a function of separation attitude but, rather, tended to occur when high separation pitch rates were observed.

These two situations bookended the desirable release conditions and provided a means to find suitable Smart Separation parameters. Both conditions included some additional padding to account for the simplicity of the modeling and instantaneous separation assumption. 


\section{Smart Release Conditions}

A major focus of the pre-test analysis was determination of the Smart Separation parameters. The Smart Separation logic requires a window of acceptable pitch angles and pitch rates. It also requires a minimum time (before which separation is inhibited) and a maximum time (when separation is commanded regardless of orientation). The logic also allows for a maximum roll angle (at which separation is commanded regardless of pitch orientation) but this constraint was not applied.

The analysis method used Monte Carlo simulations to determine the best release parameters based on pitch angle and pitch rate. As described above, a separation pitch rate of $0 \mathrm{deg} / \mathrm{s}$ allows the separation to be purely translational and simplifies the motion. Moreover, the harness geometry allowed a separation pitch angle that avoided contact between the MDS and PCDTV.

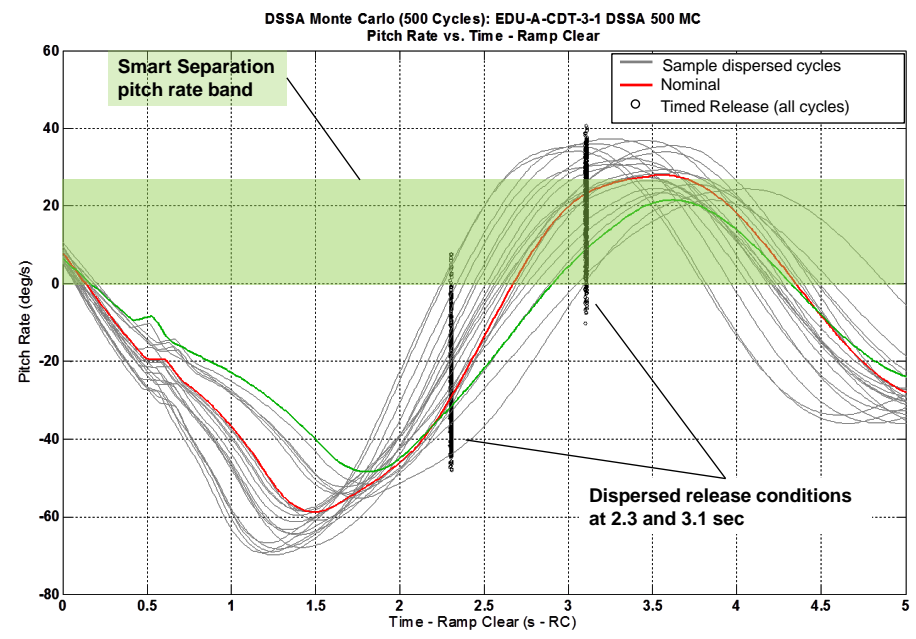

Figure 5 shows a sample Monte Carlo simulation of the extraction in which the Smart Separation time constraints have been relaxed. The small black dots indicate the various dispersed release conditions. The release does not occur in this DSSA simulation. Instead, the state vectors represented by the black dots are passed on to initialize the DSS simulation of the PCDTV alone. The spread of the release points in time is useful to note. The maximum and minimum release time of Smart Separation detection provided a starting point for determining the approriate time parameters to apply in the Smart Separation logic. Potential time constraints were then evaluated with additional Monte Carlo simulations. In general, a release at one of the time constraints indicates a shortcoming in either the avionics implementation or a shortcoming in the modeling of the extraction physics. The test team applied engineering judgment to set the time window to protect against failure while allowing the pitch and pitch rate windows to identify the optimum release point. This process is described below.

\section{Release Solution with Relaxed Time Constraints}

The MDS and riser contact limits described above provided a means to evaluate potential release conditions. To assess release conditions, a candidate set of Smart Separation parameters was applied during a Monte Carlo simulation of the extraction using DSSA. This resulted in a collection of state vectors all within the separation window but all with slightly different conditions. Using the instantaneous separation assumption, these state vectors were used to initialize a DSS Monte Carlo to simulate the programmer through touchdown phase of the test. The results of these DSS Monte Carlos were evaluated against the contact criteria described above. In general, the intent was to keep the parameter windows as broad as possible to increase the likelihood of a release on the conditions rather than on time. Potential Smart Separation windows were compared against each other by comparing the percent of cases that might have riser contact or might have PCDTV/MDS recontact. For the first PCDTV test the analysis determined that a pitch angle window between $-90 \mathrm{deg}$ and $0 \mathrm{deg}$ and a pitch rate window between $0 \mathrm{deg} / \mathrm{s}$ and $25 \mathrm{deg} / \mathrm{s}$ was the best balance. Figure 5 was produced using this type of analysis.

\section{Release Time Window Analysis}

Because this was a new technique there was uncertainty about whether the motion might be more or less dynamic than predicted or might develop slower or faster than predicted. Dispersed Monte Carlo results showed a significant spread in the time that the desired conditions occurred (see Fig. 5). In addition to the 
pitch and pitch rate windows, there is a time window included in the Smart Separation logic. Because of the large spread in time of the desired conditions seen in the Monte Carlo assessments, a concern arose that the desired release condition might occur outside the time window if it was set too tightly. On the other hand, setting the time window too broadly would leave the test vulnerable to an early release (for example, because the attitude sensors sensed a false positive release condition) or a late release (because the system failed to detect an acceptable release condition).

An analysis technique was developed to assess the affect of a commanded separation at the open or close of the window due to a failure in sensors or modeling. The technique was similar to the analysis described above. The vehicle state was extracted from DSSA Monte Carlo runs at candidate window open and closing times (ignoring the Smart Separation logic). Figure 6 shows an example of this analysis. The black dots indicate the dispersed release points for the Monte Carlo run. Note that the timed releases result in very large ranges of separation pitch rate. This is likely to result in undesirable PCDTV motion as the programmer deploys, possibly including contact between the programmer harness and the parachute compartment. These state vectors were used to initialize DSS Monte Carlos and the contact criteria were evaluated. The open and close of

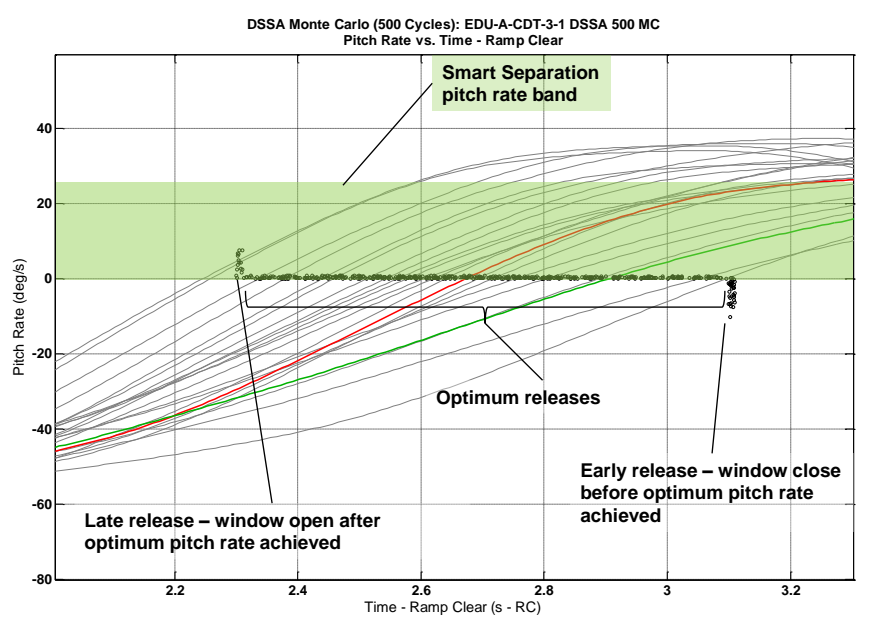
the window was adjusted until the suboptimal releases when no failure occurred were roughly balanced with the undesirable cases given that a failure occurred.

Figure 7 shows the Monte Carlo results for the release conditions when the time window is more constraining than the pitch and pitch rate constraints. The shaded band in the figure represents the pitch rate window. The small circles represent dispersed release events. Without a time constraint the releases would be expected to occur as soon as the trajectory enters the pitch rate window at $0 \mathrm{deg} / \mathrm{s}$. This is the case for the majority of the release events (labeled optimum releases). However, several cases were already within the pitch rate window when the time window opened. These are the vertical group of release points near 2.3 sec. Other cases did not enter the pitch rate window before the time window closed (and commanded separation). These are the vertical group of release points near $3.1 \mathrm{sec}$. Both of the vertical bands of release points have non-zero pitch rates which is less than optimal (assuming an appropriate pitch angle has been achieved).

This balance was revisited after each test. It was found that the method tended to constrain the time window too tightly and not allow for the actual variation and unpredictability in the motion. The result was that several early tests released at a sub-optimal (but acceptable) condition because the close of the window was set too conservatively. This was accepted in the first several tests to avoid the potential of an unacceptable release due to a Smart Release sensor or avionics failure. As confidence in the Smart Separation hardware improved with each test, the balance was adjusted to favor longer time windows to account for unpredictability in the modeling. 


\section{Test Results}

The CPAS project has executed four drop tests using the PCDTV. Each test will be summarized below.

\section{A. CDT-3-1}

Cluster Development Test 3-1 (CDT-3-1) was the first test of the PCDTV technique and was designed to test the nominal system sequence: two Drogues, three Pilots, and three Mains with two full-open Drogues used as programmers, at the low end of the dynamic pressure envelope. The PCDTV/MDS reposition maneuver occurred as planned. The smart separation system commanded PCDTV/MDS separation at the close of its time window as its combined pitch-pitch rate conditions were not achieved. As post CDT-3-2 analysis would determine, this miss can primarily be attributed to unaccounted for aircraft pitch dynamics due to its center-of-gravity change as the mated vehicle was being extracted. The pretest simulated pitch profile was defined for a baseline C-130 aircraft, not the stretched L383G variant that was actually flown, and the result was pitch plane conditions at the aircraft exit that were outside of preflight Monte Carlo predictions. In spite of the non-optimal pitch conditions, the separation was successful and the parachute sequence deployed nominally.

\section{B. CDT-3-2}

Cluster Development Test 3-2 (CDT-3-2) was designed to test performance of the system with the complete failure of a single Main parachute. The test was performed at the low end of the dynamic pressure envelope with two Drogues reefed at $48 \%$ as Programmers, two Drogues, two Pilots, and two Mains. As experienced in CDT-3-1, separation was commanded on the expiration of the time window and the PCDTV was released with an unexpected negative pitch rate which threatened to rotate the parachute compartment end forward. However, the programmer parachutes, after inflating directly to their reefed condition via static line deployment from the MDS, arrested the pitch rate and the separation was successful and the EDU parachute sequence deployed nominally. The deficiency in the C-130 aircraft pitch model was addressed in CDT-3-4 preflight analysis.

\section{CDT-3-4}

Cluster Development Test 3-4 (CDT-3-4) was designed to test performance of the system when one of the Main parachutes skips the second reefing stage. The test was performed at the low end of the dynamic pressure envelope with two Drogues, three Pilots, and three Mains. One Drogue reefed at 48\% was used as the programmer.

Reconstructed pitch profiles from CDT-3-1 and CDT-3-2 were used to disperse preflight Monte Carlo simulations. However, those tests used a stretched L383G variant rather than the baseline C-130A used in CDT-3-4, and the change is believed to be the reason that the measured CDT-3-4 pitch rate was higher than the maximum prediction at ramp clear. The Smart Separation pitch angle criterion was met as the minimum time threshold was reached with an acceptable pitch value. Pitch rate entered its acceptable range just over $0.3 \mathrm{sec}$ prior to the close of the window, and separation was commanded, and the maneuver was nominal, resulting in a very stable PCDTV trajectory. These results validated a pre-flight decision to expand the time dimension of the window despite preflight Monte Carlo analysis which had indicated that a much shorter window would suffice. The EDU parachute systems deployed and performed as planned.

\section{CDT-3-6}

Cluster Development Test 3-6 (CDT-3-6) was designed to test the nominal Drogue, Pilot, and Main sequence at a higher dynamic pressure than the previous tests, in the middle of the Drogue deployment envelope. The extraction was performed with two 28 -ft parachutes (due to heavier mated vehicle weight) as opposed to a single extraction parachute used on previous tests. Because there were two extraction parachutes, the total load passing through the harness legs was twice that of previous tests. This caused the spreader bar to bend during the reposition. Fortunately, the damage of this component did not negatively affect the test. This problem was corrected with a stronger component future tests. Similar to CDT-3-4, separation occurred on design pitch-pitch rate conditions. The resulting PCDTV trajectory was very stable despite the tightly reefed programmer parachute. 


\section{Conclusion}

The PCDTV has become the workhorse of the CPAS EDU test series and has demonstrated numerous parachute deployments at a range of dynamic pressures. The preliminary design of the separation event was difficult but the analysis technique has been easily adaptable to modest changes in the test profile. Design of the extraction and separation sequence can now be performed in a matter of days rather than weeks.

\section{Acknowledgments}

Keith Anderson of ATK, Pete Cuthbert of NASA, and Peter Schulte of Jacobs Engineering performed significant analyses that were instrumental in bringing the PCDTV technique to test readiness and also made continued improvements to the prediction capability. The authors also wish to thank Ken Nguyen of Jacobs Engineering for supplying many of the diagrams of the PCDTV used in this paper. There were many other hardware, avionics, and operations engineering tasks that are not discussed in this paper. These challenges were met by the very capable CPAS team who also deserve credit for the success of the tests. Finally, a special thanks goes to Pat Galvin and the CPAS Analysis team for assistance and review in writing this paper.

\section{References}

${ }^{1}$ Machin, R. A., Stewart, C. E., Evans, C. T., and McMichael, J. H., "Human Rating the Orion Parachute System," 21st AIAA Aerodynamics Decelerator Systems Technology Conference, Dublin, Ireland, May 2011, AIAA 2011-2502.

${ }^{2}$ Morris, A. L., Fraire, U., Bledsoe, K. J., Moore, J. W., Olson, L. M., and Ray, E. S., "Simulating New Drop Test Vehicles and Test Techniques for the Orion CEV Parachute Assembly System," 21st AIAA Aerodynamics Decelerator Systems Technology Conference, Dublin, Ireland, May 2011, AIAA 2011-2616.

${ }^{3}$ Ray, E. S. and Morris, A. L., "Challenges of CPAS Flight Testing," 21st AIAA Aerodynamics Decelerator Systems Technology Conference, Dublin, Ireland, May 2011.

${ }^{4}$ King, R., Hengel, J. E., and Wolf, D., "Ares Decelerator System Heavy Air Drop Test Program," 21st AIAA Aerodynamics Decelerator Systems Technology Conference, Dublin, Ireland, May 2011, AIAA 2011-2504.

${ }^{5}$ Moore, J. W. and Morris, A. L., "Development of a Smart Release Algorithm for Mid-Air Separation of Parachute Test Articles," 21st AIAA Aerodynamics Decelerator Systems Technology Conference, Dublin, Ireland, May 2011, AIAA $2011-2602$.

${ }^{6}$ Cuthbert, P. A., "A Software Simulation of Cargo Drop Tests," 17th AIAA Aerodynamic Decelerator Systems Technology Conference, Monterey, California, May 2003, AIAA 2003-2132.

${ }^{7}$ Moog, R. D., Parachute Simulation Userś Guide Computer Program UD233A, Martin Marietta Corp., Denver Aerospace Division, Denver, Colorado, USA, Feb. 1986.

${ }^{8}$ Cuthbert, P. A., "A Desktop Application to Simulate Cargo Drop Tests," 18th AIAA Aerodynamic Decelerator Systems Technology Conference and Seminar, Munich, Germany, May 2005, AIAA 2005-1623.

${ }^{9}$ Moore, J. W., "A Boilerplate Capsule Test Technique for the Orion Parachute Test Program," 22nd AIAA Aerodynamics Decelerator Systems Technology Conference, Daytona Beach, Florida, March 2013, Submitted for Publication. 